\title{
Are acute asthma guidelines being adhered to? A review of practice in a tertiary hospital
}

\author{
S Hoosen, MB ChB, FC Paed (SA), Dip Allerg (SA), MMed (Paed), DCH (SA); \\ V Naidoo, MB BCh, FC Paed (SA), Dip Allerg (SA), Cert Pulm Paed (SA); \\ R Masekela, MB BCh, MMed (Paed), Dip Allerg (SA), Cert Pulm Paed (SA), FCCP, PhD \\ Department of Paediatrics and Child Health, Nelson R Mandela School of Medicine, University of KwaZulu-Natal, Durban, South Africa
}

Corresponding author: S Hoosen (safiahoosen@gmail.com)

\begin{abstract}
Background. Asthma is the most common chronic disease of childhood and presents a diagnostic and management dilemma to many healthcare practitioners. The use of guidelines in managing paediatric acute asthma is essential to improve quality of life and reduce morbidity and mortality.

Objective. To review the adherence of medical practitioners to the South African (SA) guideline for the management of acute asthma in children.

Methods. A one-year retrospective chart review was undertaken at the King Edward VIII Hospital in Durban, SA, in 2014. Asthmatic patients younger than 12 years who presented to the paediatric resuscitation unit were included. Demographic data, clinical parameters and the initial management of the asthma exacerbations at presentation were recorded and compared with the recommendation of the SA guideline (2013). Any deviation from the guideline was regarded as a lack of adherence to the protocol.

Results. A total of 39 cases were reviewed, of which 24 (61.5\%) were male. The severity of the asthma exacerbation was unclassified in the majority of cases $(92.3 \%)$. Deviations from the recommended protocol included the administration of supplemental oxygen in 6 cases, chest radiography in 22 cases (56.4\%) when it was not indicated and unnecessary antibiotic use in 10 cases (25.6\%). All patients received $\beta_{2}$-agonists via an oxygen-driven nebuliser.

Conclusion. Adherence to the current acute asthma guideline was poor in this study setting. Both imaging and antibiotics were used unnecessarily, which are associated with inherent risks of cancer and antibiotic resistance, respectively.
\end{abstract}

S Afr J Child Health 2020;14(3):119-123. https://doi.org/10.7196/SAJCH.2020.v14i3.1658

Asthma is a serious global health problem affecting all age groups. Its prevalence is increasing in many countries, especially among children. ${ }^{[1]}$ Asthma is the most common chronic disease of childhood and the leading cause of childhood morbidity from chronic disease as measured by school absences, emergency department visits and hospitalisations. ${ }^{[2]}$

In a multinational study across 16 countries, the prevalence of asthma and asthma symptoms was shown to be high (20.3\%) in a South African (SA) cohort of children in Cape Town. ${ }^{[3]}$ The appropriate management of acute asthma exacerbations is therefore essential to improve quality of life and to reduce both direct and indirect costs.

Guidelines for the management of diseases are available to primary care physicians to improve outcomes and minimise complications. The SA guideline for the management of acute asthma in children was revised in 2013, prompted by factors such as changes in international guidelines, updates on the assessment of acute severe asthma, the need for different treatment strategies in young children compared with older children, and the development of new asthma therapies. ${ }^{[4]}$ The revised set of guidelines provides protocols for managing asthmatic children presenting with acute exacerbations, first by ensuring correct classification of exacerbation severity and then guidance on the appropriate therapy.

With 10.9 million people (19.9\%), KwaZulu-Natal (KZN) has the second largest population in SA (19.9\% of an estimated national population of 54.96 million). ${ }^{[5]}$ Approximately $30.2 \%$ of the total population is younger than 15 years, with about 3.8 million (22.9\%) of these children living in $\mathrm{KZN}^{[5]}$ A large number of the people in KZN reside in rural areas, where biomass fuels, paraffin lamps, wood, candles and liquefied petroleum gas are used for common household purposes such as cooking, heating water and to provide lighting. ${ }^{[6]}$ Poor asthma control and increased exacerbations are linked to a lack of access to a diagnosis and appropriate treatment and also to exposure to indoor and outdoor pollution. In a study conducted in the southern areas of Durban, SA, to assess the associations between pollution and respiratory outcomes in schoolchildren, it was found that children from industrially exposed communities experienced a higher prevalence of persistent asthma and marked airway hyperreactivity than those living far from industrial sources. ${ }^{[7]}$

The aim of our study was to review treating physicians' adherence to the guideline for the management of acute asthma in children presenting with acute asthma exacerbations to an emergency paediatric unit in a regional hospital in Durban, SA.

\section{Methods \\ Study setting}

A one-year retrospective chart review was conducted at the King Edward VIII Hospital (KEH) in Durban, SA, between 1 January and 31 December 2014. KEH is one of the largest regional hospitals in $\mathrm{KZN}$, with 852 beds and a paediatric resuscitation unit (PRU) that also serves as the paediatric emergency admission unit. The PRU has 17 beds and admits, on average, nine children per day for various acute illnesses, including asthma. This unit is equipped with two ventilators and can offer interim respiratory support to patients until their transfer to an intensive care unit. The unit admits patients from the paediatric outpatient department, local clinics and nearby 
district hospitals. It is staffed by nurses, junior medical interns, junior paediatric registrars and paediatric specialists.

\section{Study population}

All cases of children between the age of 3 months and 12 years with an admission diagnosis of acute asthma exacerbation were included in the study. The pre-existing diagnosis of asthma was accepted whether it was documented (i.e. based on the doctor's diagnosis, previous discharge summaries or clinic notes) or verbal (i.e. based on communication from a hospital, clinic or general practitioner). The case management notes were reviewed to assess the physician's management of each case depending on the severity of the episode (mild, moderate or severe exacerbation). The treatment was then compared with the management protocol recommendations. ${ }^{[4]}$ Any deviation from the protocol was noted and recorded as nonadherence.

Case files of patients with an alternative diagnosis on further enquiry and those with hyper-reactive airways disease or viralinduced wheezing were excluded from the analysis, as were undocumented patients and those with missing charts.

Demographic data collected included gender, ethnicity, physical address and nutritional status. Asthma severity was recorded based on the classification of the SA guideline ${ }^{[4]}$ and the management strategy was noted, including the use of supplemental oxygen, short-acting $\beta_{2}$-agonists (SABAs), arterial blood gas (ABG) measurements, chest X-rays (CXR), steroid therapy and antibiotics. Clinical parameters noted were pulse rate, respiratory rate, use of accessory muscles of respiration, wheezing, level of consciousness and oxygen saturation $\left(\mathrm{SpO}_{2}\right)$ in room air.

The different asthma exacerbations were categorised as follows based on the SA guideline: ${ }^{[4]}$

1. Mild exacerbations: Those just outside the normal range of variation for an individual patient.

2. Moderate exacerbations: Increasing symptoms, worsening lung function and/or increased rescue bronchodilator use for at least 2 days without the need for systemic steroids.

3. Severe exacerbations: Symptoms of severe asthma (breathlessness, inability to complete sentences or feed, use of accessory respiratory muscles, tachycardia, tachypnea, $\mathrm{SpO}_{2}<92 \%$, decreased peak expiratory flow rate), together with presentation to a hospital and treatment with a systemic steroid for at least 3 days.

4. Acute severe asthma: Severe asthma unresponsive to repeated courses of $\beta_{2}$-agonist therapy.

5. Near-fatal asthma: Acute severe asthma associated with a respiratory arrest or hypercarbia.

The SA acute asthma guideline advises the following: ${ }^{[4]}$

- All patients should have clinical parameters and a pulse oximetry reading recorded, and should receive supplemental oxygen for $\mathrm{SpO}_{2}<92 \%$ in room air.

- Inhaled $\beta_{2}$-agonists should be delivered by a pressurised metereddose inhaler (pMDI) with a spacer (2 - 10 puffs, each inhaled separately, with five tidal breaths at intervals of 15 - 30 seconds) or by an oxygen-driven nebuliser. A pMDI with a spacer is the preferred drug delivery device for the treatment of mild to moderate acute asthma, whereas oxygen-driven nebulisers are preferred for severe or life-threatening acute asthma.

- $A B G$ measurements are indicated only in severe or life-threatening asthma not responding to treatment.

- Indications for chest radiography are few and specific (failure to respond to standard therapy, clinical signs suggestive of a lung collapse or pneumothorax, life-threatening asthma unresponsive to maximal therapy or a need to exclude an alternative diagnosis).

- Steroids should be administered early in the acute attack. Oral steroids are as effective as intravenous treatment and are preferred for their ease of administration.

- Antibiotics are not routinely indicated in acute asthma, which is usually precipitated by a viral infection.

\section{Data analysis}

The Statistical Package for Social Sciences version 22 (IBM Corp., US) was used for statistical analysis. Descriptive statistics (frequencies and percentages) were used to summarise the results for categorical data (gender, ethnicity, nutrition, diagnosis and classification), the use of oxygen, antibiotics and adjunctive investigations such as ABG and CXR. Independent-samples $t$-tests were used to analyse the classification and to assess whether correct SABA use affected the duration of hospital stay. Confidence intervals (CIs) were calculated for each $t$-test. A significance level of $p<0.05$ was used.

\section{Ethical considerations}

Ethical approval was obtained from the Biomedical Research Ethics Committee of the University of KZN prior to commencement of the study (ref. no. BE385/15). Permission was also obtained from the KZN Department of Health and the medical manager of KEH. As the study did not involve direct patient contact, individual informed consent was not required.

\section{Results}

Of the 159 patient records screened, 39 cases were eligible for inclusion in the study (Fig. 1). The mean age of the patients was 69 months, with a male preponderance of $61.5 \%$. Of the 39 cases included, 36 patients were black, 2 were white and 1 was Indian. All the patients were recorded to have normal nutrition, with a weightfor-height $z$-score of between 0 and +2 standard deviations when plotted on the World Health Organization growth charts. ${ }^{[8]}$ The clinical diagnosis of asthma was documented in 27 cases, while 12 cases had a verbal diagnosis.

The severity of the exacerbation was not classified in 36 cases (92.3\%). Of the remaining cases, one was classified as mild and two as acute severe asthma. Signs of respiratory distress were recorded in all 39 cases, wheezing in 38 cases and the level of consciousness in 34 cases.

ABG measurement was performed in four cases, although it was indicated only in three. Chest radiography was performed in 30 cases $(76.9 \%)$, although in 22 cases $(73.3 \%)$ it was not indicated (Fig. 2).

Supplemental oxygen was administered in 11 cases, although hypoxia $\left(\mathrm{SpO}_{2}<92 \%\right)$ was recorded in only five cases; in one case, supplemental oxygen was not given although it was indicated. All of the patients received $\beta_{2}$-agonists via an oxygen-driven nebuliser. SABAs were administered at intervals of $20-30$ minutes in 28 patients $(71.8 \%)$ and at longer intervals in 11 patients. All of the patients received steroids: via the oral route in 38 patients (97.4\%), at a dose of $1-2 \mathrm{mg} / \mathrm{kg}$, and intravenously in one patient. Steroids were used for less than 3 days in 28 patients and for 3 - 5 days in 11 patients.

Antibiotics were administered in 14 patients (35.9\%), although this was not indicated in 10 of these patients $(71.4 \%)$. The mean (standard deviation) duration of hospital stay was 2.2 (1.4) days. There was no statistically significant difference between the classification of asthma exacerbation severity and length of hospital stay $(p=0.35 ; 95 \%$ CI $1.7-2.7)$. 


\section{Discussion}

Our review showed that the severity of asthma exacerbation was unclassified in more than $90 \%$ of cases. There was evidence of potentially harmful therapies being used when not indicated, with chest radiography in almost three-quarters of the patients, high levels of antibiotic use in over a third and supplemental oxygen being administered unnecessarily in over half of the cases. The deviations from the recommended guidelines did not affect short-term outcomes, with length of stay appearing to be independent of accurate disease classification and disease management. However, appropriate use of oral corticosteroids was noted, with the associated duration of therapy being in accordance with the recommended guidelines. Prolonged courses of corticosteroid therapy were avoided owing to the effect on growth, particularly in young children. Appropriate assessments for respiratory failure were conducted when necessary.

In the emergency department, the severity of an asthma exacerbation determines the intensity of treatment and the frequency of patient monitoring. ${ }^{[9]}$ Unclassified exacerbations can negatively affect patient morbidity and mortality, as seriously ill patients could go unrecognised. Severe exacerbations of asthma are potentially lifethreatening and therefore require prompt care, close observation for deterioration and frequent treatment. ${ }^{[9]}$ Health practitioners were studious in their recording of the clinical parameters of respiratory distress, likely owing to the use of a provincial admission tool that makes recording of such information mandatory. However, this did not result in the overall assessment of asthma exacerbation severity. Such an assessment is important, as it guides the decision for

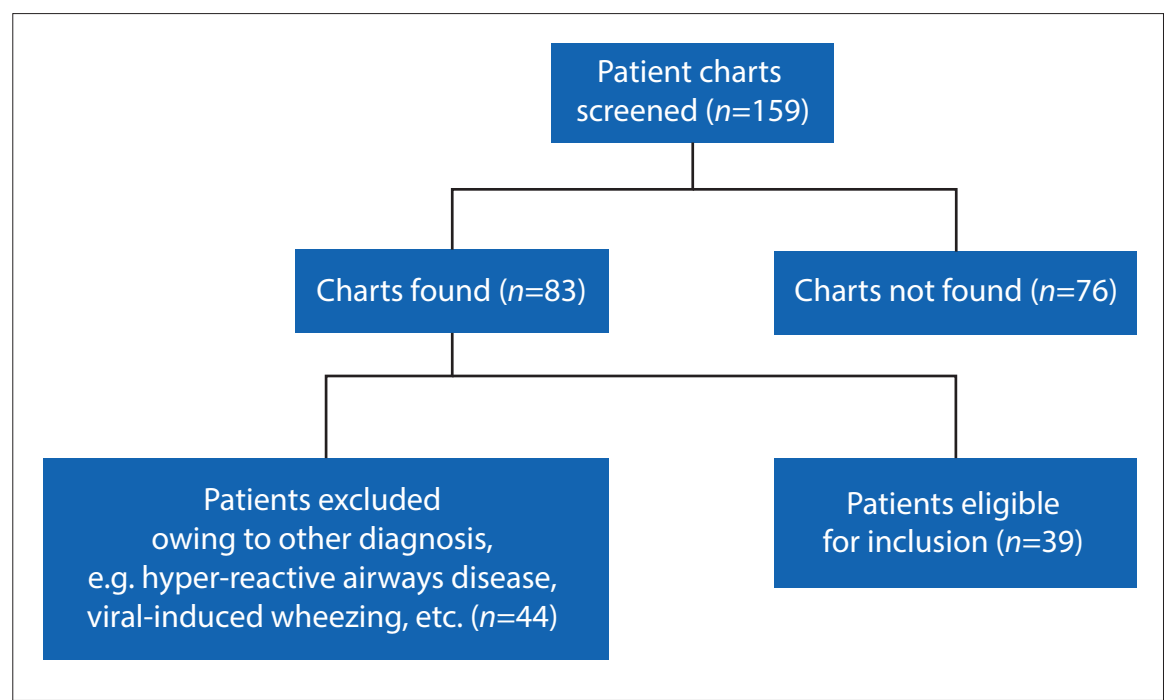

Fig. 1. Selection of study sample.

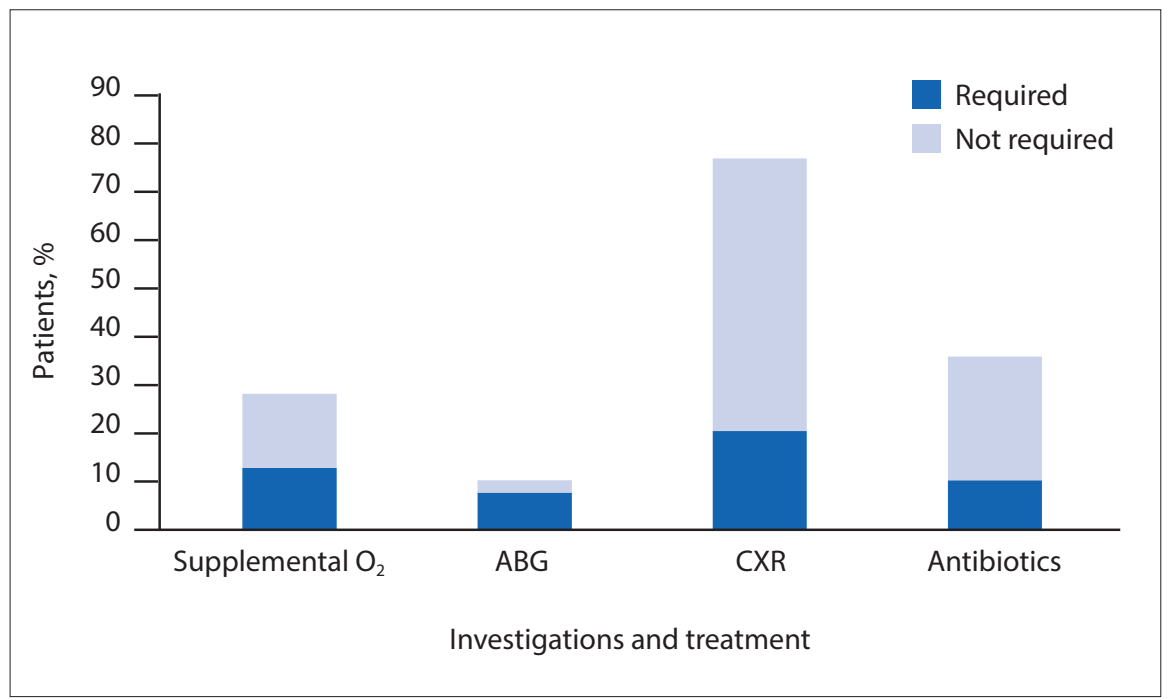

Fig. 2. Use of adjunctive investigations and therapy. ( $A B G=$ arterial blood gases; $C X R=$ chest $X$-rays. $)$

subsequent investigations and treatment to reduce the risk of asthma-related deaths.

Treatment of asthma exacerbation is aimed at rapidly relieving airflow obstruction and hypoxaemia and preventing relapses. ${ }^{[1]}$ Hypoxaemia is known to cause pulmonary hypertension, although the exact severity and duration of hypoxaemia needed to do this are unknown. ${ }^{[10]}$ The factors affecting individual susceptibility are also unknown. ${ }^{[10]}$ Although hypoxaemia is detrimental to a patient's condition, supplemental oxygen should also be used judiciously. In the current study, supplemental oxygen was administered unnecessarily in several cases. There is strong evidence that an imbalance between the reducing and oxidising systems exists in asthma, with a more oxidative state being favoured. ${ }^{[11]}$ Endogenous and exogenous reactive oxygen species have a major role in airway inflammation and are determinants of asthma severity. ${ }^{[11]}$ Asthma is also associated with decreased antioxidant defences. ${ }^{[11]}$ At low levels of oxidative stress, antioxidants can restore cellular redox homeostasis. ${ }^{[11]}$ However, at high levels of oxidative stimuli, additional sets of intracellular signalling cascades are triggered, which are potentially proinflammatory. ${ }^{[11]}$ These pro-inflammatory factors can both worsen an acute asthmatic exacerbation and prolong the overall time to recovery, and therefore the unnecessary use of oxygen should be curbed.

SABAs are the mainstay of therapy for acute asthma and the recommended first-line treatment. ${ }^{[4]}$ The Global Initiative for Asthma (GINA) guideline reiterates that in patients presenting with an asthmatic exacerbation, treatment should be started with repeated administrations of SABAs. ${ }^{[1]}$ A review assessing the use of SABAs for emergency management of childhood asthma found that when SABAs were used as first-line therapy, hospital admission decreased by $44 \%$ in younger children and length of stay in the emergency department decreased by 33 minutes in older children. ${ }^{[12]}$

In our study, SABAs were used at the correct dosing intervals in the majority of patients. Although only patients with acute severe asthma should receive $\beta_{2}$-agonists nebulised with oxygen, ${ }^{[13]}$ all of the patients in our study received SABAs via an oxygendriven nebulised system.

The prolonged use of steroids in children has been associated with detrimental longterm effects, namely adrenal suppression, immune suppression, osteopenia and a decreased overall height velocity. Limiting the duration of steroid therapy to need is therefore important to lessen the negative 
long-term effects. Short courses of oral steroids (1 - $2 \mathrm{mg} / \mathrm{kg}$ prednisolone or prednisone for 3 - 5 days) are not associated with adrenal or immune suppression, even when used on several occasions over the course of 1 year. ${ }^{[14]}$ In the current study, oral steroids were used for appropriately short durations and at the correct doses. Children with moderate to severe asthma exacerbations should receive systemic steroids as early as feasible as part of their initial treatment. ${ }^{[15]}$ There is no known advantage of using higher doses of corticosteroids in severe asthma exacerbations, nor is there any advantage of intravenous administration over oral therapy, provided that gastrointestinal transit time or absorption is not impaired. ${ }^{\left[{ }^{[3}\right.}$

Children are particularly vulnerable to radiation risks. ${ }^{[16]}$ Radiation risks are expected to be higher because of the higher proportion of dividing cells in younger individuals and also because of the longer lifespan available for a potential cancer to be expressed. ${ }^{[17]}$ The inappropriate use of chest radiography in more than half of the patients in this study poses a persistent lifetime risk for the development of radiation-related cancer. Numerous epidemiologic cohort studies of childhood exposure to radiation for treatment of benign diseases have demonstrated radiationrelated risks of developing cancer of the thyroid, breast, brain and skin, as well as leukaemia. ${ }^{[18]}$ These studies have shown that risks of radiation-related cancer are greatest for those exposed early in life, and these risks appear to persist throughout life. ${ }^{[18]}$ The mean age of these children was $0.5-7.8$ years, ${ }^{[18]}$ compared with the mean age range of 5.8 years for the children in our study. Indications for chest radiography are few and specific and should therefore be used judiciously, bearing in mind the cumulative risk acquired in children affected by chronic diseases such as asthma when they are subjected to routine chest X-rays.

Antibiotics were used unnecessarily in more than a quarter of the current cohort. Antibiotics are not recommended for the treatment of acute asthma exacerbations, except if needed to treat comorbid conditions (e.g. in patients with fever and purulent sputum, when there is evidence of pneumonia, or in the case of suspected bacterial sinusitis). ${ }^{[19]}$ In a study to assess the role of viral infections in asthmatic exacerbations, it was found that viral infections were associated with $80-85 \%$ of asthmatic exacerbations in schoolaged children. ${ }^{[20]}$ Rhinoviruses accounted for two-thirds of these infections, with coronaviruses being the next most common. ${ }^{[20]}$ Antibiotics are therefore ineffective, as viruses trigger asthmatic exacerbations more often than bacteria. Principles of antibiotic stewardship are of utmost importance to mitigate antimicrobial resistance.

In a study that assessed adherence to a clinical practice guideline for acute asthma in a paediatric emergency department, it was found that $68 \%$ of patients were managed with complete adherence to the guideline. ${ }^{[21]}$ In a large multicentre Swedish study on the management of acute asthma in children, clinical examination and non-pharmacological treatment had the lowest adherence levels. ${ }^{[22]}$ The challenge of adhering to guidelines, as also seen in our study, is a worldwide phenomenon.

\section{Strengths and limitations of the study}

A strength of the study is that it was performed in a regional hospital where adequate resources are available to review guideline adherence patterns. Limitations of the study include the small cohort of patients, due largely to the number of missing charts. However, despite the relatively small size, the study is considered to have provided valuable insight by demonstrating low adherence patterns despite the availability of adequate resources. Long-term follow-up of these patients to assess the effects of their in-hospital management was not included. Finally, the presence of dual systems, with different national and provincial guidelines, results in poor clinician adherence to guideline therapies and in physicians 'picking' which protocol to use.

It is recommended that both provincial and national protocols be streamlined. Adherence to the recommended guidelines could be improved by conducting training seminars with the junior medical staff at the start of their paediatric rotation, followed by an internal audit and peer review to assist in identifying deficits. Guideline posters should also be displayed in the PRU for ease of reference.

\section{Conclusion}

The review showed poor adherence to the current acute asthma guidelines, with inappropriate use of both imaging and antibiotics noted in young children. This increases the risk of radiationrelated cancer and drives antibiotic resistance, respectively. Medical practitioners' adherence to guidelines could lessen the problems identified in this study and contribute to an improved quality of life for asthmatic children.

Declaration. This manuscript was submitted in partial fulfilment of the requirements for a MMed (Paeds) degree.

Acknowledgements. The authors acknowledge the KwaZulu-Natal Department of Health and the King Edward VIII Hospital for approval of the study, and thank Dr Shashikant Ramji for the use of the PRU data and Ms Fikile Nkwanyana for assistance with statistical analysis.

Author contributions. SH, RM and VN all contributed to study conceptualisation. SH was responsible for data collection and was supported in data analysis and manuscript development by RM.

Funding. None.

Conflicts of interest. None.

1. FitzGerald JM, Bateman ED, Boulet LP, et al. Global strategy for asthma management and prevention. Fontana, WI: Global Initiative for Asthma, 2015 ginasthma.org/wp-content/uploads/2016/01/GINA_Report_2015_Aug11-1. pdf (accessed 17 September 2015).

2. Masoli M, Fabian D, Holt S, Beasley R, Global Initiative for Asthma (GINA) Program. The global burden of asthma: Executive summary of the GINA Dissemination Committee report. Allergy 2004;59(5):469-478. https://doi. org/10.1111/j.1398-9995.2004.00526.x

3. Ait-Khaled N, Odhiambo J, Pearce N, et al. Prevalence of symptoms of asthma, rhinitis and eczema in 13- to 14-year-old children in Africa: The International Study of Asthma and Allergies in Childhood Phase III. Allergy 2007;62(3):247258. https://doi.org/10.1111/j.1398-9995.2007.01325.x

4. Kling S, Zar HJ, Levin ME, et al. Guideline for the management of acute asthma in children: 2013 update - Part 3. S Afr Med J 2013;103(3):199-207. https://doi. org/10.7196/SAMJ.6658

5. Statistics South Africa. Mid-year population estimates. 23 July 2015. Statistical Release P0302. Pretoria: SSA, 2015. https://www.statssa.gov.za/publications/ P0302/P03022015.pdf (accessed 12 May 2016).

6. Aitken R. Household energy use: A comparison of household energy consumption and expenditure across three provinces. J Energy S Africa 2007;18(1):20-28. https://doi.org/10.17159/2413-3051/2007/v18i1a3338

7. Naidoo RN, Jack C, Robins TG, Batterman S, Mentz G. Ambient pollution and respiratory outcomes among schoolchildren in Durban, South Africa. S Afr J Child Health 2013;7(4):127-134. https://doi.org/10.7196/sajch.598

8. World Health Organization. WHO Child Growth Standards. http://www.who.int/ childgrowth/standards/weight_for_length_height/en/(accessed 10 August 2018).

9. Camargo Jr CA, Rachelefsky G, Schatz M. Managing asthma exacerbations in the emergency department: Summary of the National Asthma Education and Prevention Program Expert Panel Report 3 guidelines for the management of asthma exacerbations. Proc Am Thorac Soc 2009;6(4):357-366. https://doi. org/10.1016/j.jemermed.2009.06.105

10. Balfour-Lynn IM, Field DJ, Gringras P, et al. BTS guidelines for home oxygen in children. Thorax 2009;64(Suppl 2):ii1-ii26. https://doi.org/10.1136/ thx.2009.116020

11. Sahiner UM, Birben E, Erzurum S, Sackesen C, Kalayci O. Oxidative stress in asthma. World Allergy Organ J 2011;4(10):151-158. https://doi.org/10.1097/ WOX.0b013e318232389e 
12. Pollock M, Sinha IP, Hartling L, Rowe BH, Schreiber S, Fernandes RM. Inhaled short-acting bronchodilators for managing emergency childhood asthma: An overview of reviews. Allergy 2017;72(2):183-200. https://doi.org/10.1111/ all.13039

13. Inwald D, Roland M, Kuitert L, McKenzie SA, Petros A. Oxygen treatment for acute severe asthma. BMJ 2001;323(7304):98-100. https://doi.org/10.1136/ bmj.323.7304.98

14. Rieder MJ. The child with multiple short courses of steroid therapy. Paediatr Child Health 2003;8(4):226. https://doi.org/10.1093/pch/8.4.226

15. Ortiz-Alvarez O, Mikrogianakis A. Managing the paediatric patient with an acute asthma exacerbation. Paediatr Child Health 2012;17(5): 251-256. https:// doi.org/10.1093/pch/17.5.251

16. Kirk R. Radiation exposure in children. J Heart Lung Transplant 2014;33(11):1117-1118. https://doi.org/10.1016/j.healun.2014.08.007

17. Brenner DJ, Doll R, Goodhead DT. Cancer risks attributable to low doses of ionizing radiation: Assessing what we really know. Proc Natl Acad Sci USA 2003;100(24):13761-13766. https://doi.org/10.1073/pnas.2235592100

18. Kleinerman RA. Cancer risks following diagnostic and therapeutic radiation exposure in children. Pediatr Radiol 2006;36(2):121-125. https://doi. org/10.1007/s00247-006-0191-5
19. National Institutes of Health. Guidelines for the Diagnosis and Management of Asthma: Update on Selected Topics 2002. Bethesda, MD: NIH, 2002.

20. Johnston SL, Pattemore PK, Sanderson G, et al. Community study of role of viral infections in exacerbations of asthma in 9-11 year old children. BM] 1995;310(6989):1225-1229. https://doi.org/10.1136/bmj.310.6989.1225

21. Scribano PV, Lerer T, Kennedy D, Cloutier MM. Provider adherence to a clinical practice guideline for acute asthma in a pediatric emergency department. Acad Emerg Med 2001;8(12):1147-1152. https://doi.org/10.1111/j.1553-2712.2001. tb01131.x

22. Jonsson M, Egmar AC, Kiessling A, et al. Adherence to national guidelines for children with asthma at primary health centres in Sweden: Potential for improvement. Prim Care Respir J 2012;21(3):276-282. https://doi.org/10.4104/ pcrj.2012.00051

Accepted 8 January 2020 